\title{
Effects of One-step and Two-step Treatment Regimes on the Characteristics of Plasma Electrolytic Oxidation (PEO) Coatings on Aluminum
}

\author{
Ye-kang Wu ${ }^{l}$, Hui-ping Han ${ }^{l}$, Rui-qiang Wang ${ }^{2}$, Guo-rui Wu ${ }^{1,3}$, Xu-zhen Zhang ${ }^{l}, Y u \mathrm{Su}^{l}$, \\ Da-long Lit, Chang-hong Guo ${ }^{4}$, Sheng-xue Yu ${ }^{1}$, De-jiu Shen ${ }^{1, *}$, Philip Nash ${ }^{5}$ \\ ${ }^{1}$ State Key Laboratory of Metastable Materials Science and Technology, College of Materials Science \\ and Engineering, Yanshan University, Qinhuangdao 066004, PR. China \\ ${ }^{2}$ School of Material Science and Engineering, Beijing Institute of Technology, Beijing 100081, PR. \\ China \\ ${ }^{3}$ CITIC Dicastal Limited by Share Ltd, Qinhuangdao 066000, PR. China \\ ${ }^{4}$ College of Mechanical Engineering, Yanshan University, Qinhuangdao 066004, PR. China \\ ${ }^{5}$ Thermal Processing Technology Center, Illinois Institute of Technology, Chicago, IL 60616, USA \\ *E-mail: DejiuShen@163.com
}

doi: $10.20964 / 2019.05 .19$

Received: 25 November 2018 / Accepted: 25 January 2019 / Published: 10 April 2019

\begin{abstract}
Plasma electrolytic oxidation (PEO) processing of aluminum is galvanostatically carried out through one-step and two-step treatment regimes in an alkaline sodium silicate electrolyte without and with sodium tungstate additive agent. The microstructure, phase composition and corrosion resistance of the resultant coatings are investigated by means of scanning electron microscopy (SEM), X-ray diffraction (XRD), 3-dimensional imaging techniques and potentiodynamic polarization tests. Some PEO coatings are stripped from the aluminum substrate via an electrochemical coating stripping method to intuitively disclose the corresponding relations among the characteristic structures of the free surface, fractured cross-section and coating/substrate interface. Further, coating growth models describing the one-step and two-step treatments are proposed according to the present PEO coating microstructure characteristics. The potentiodynamic polarization test results demonstrate that the one-step PEO coating treated in the compound silicate-tungstate electrolyte for $20 \mathrm{~min}$ exhibits the best resistance to corrosion as compared to bare $\mathrm{Al}$ and other PEO coatings.
\end{abstract}

Keywords: Plasma electrolytic oxidation; Treatment regime; Stripped coating; Microstructure corresponding relation; Growth model 
(C) 2019 The Authors. Published by ESG (www.electrochemsci.org). This article is an open access article distributed under the terms and conditions of the Creative Commons Attribution license (http://creativecommons.org/licenses/by/4.0/). 